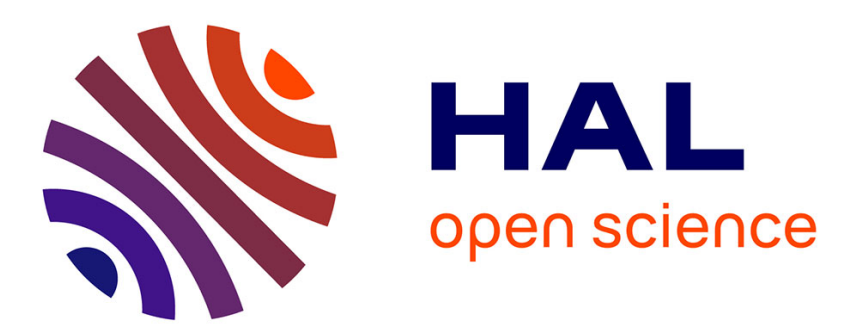

\title{
An Additive Schwarz Method Type Theory for Lions's Algorithm and a Symmetrized Optimized Restricted Additive Schwarz Method
}

\author{
Ryadh M Haferssas, Pierre Jolivet, Frédéric Nataf
}

\section{- To cite this version:}

Ryadh M Haferssas, Pierre Jolivet, Frédéric Nataf. An Additive Schwarz Method Type Theory for Lions's Algorithm and a Symmetrized Optimized Restricted Additive Schwarz Method. SIAM Journal on Scientific Computing, 2017, 39 (4), pp.A1345 - A1365. 10.1137/16M1060066 • hal-01278347

\section{HAL Id: hal-01278347 \\ https://hal.science/hal-01278347}

Submitted on 24 Feb 2016

HAL is a multi-disciplinary open access archive for the deposit and dissemination of scientific research documents, whether they are published or not. The documents may come from teaching and research institutions in France or abroad, or from public or private research centers.
L'archive ouverte pluridisciplinaire HAL, est destinée au dépôt et à la diffusion de documents scientifiques de niveau recherche, publiés ou non, émanant des établissements d'enseignement et de recherche français ou étrangers, des laboratoires publics ou privés. 


\title{
An additive Schwarz method type theory for Lions' algorithm and Optimized Schwarz Methods
}

\author{
Ryadh Haferssas ${ }^{* 1}$, Pierre Jolivet ${ }^{\dagger 2}$, and Frédéric Nataf ${ }^{\ddagger 1}$ \\ ${ }^{1}$ Laboratory J.L. Lions, UPMC, CNRS UMR7598 and INRIA \\ Team Alpines, France \\ ${ }^{2}$ Toulouse Institute of Computer Science Research, France
}

4 février 2016

\begin{abstract}
Résumé
Optimized Schwarz methods (OSM) are very popular methods which were introduced by P.L. Lions in 27] for elliptic problems and by B. Després in 8 for propagative wave phenomena. We give here a theory for Lions' algorithm that is the genuine counterpart of the theory developed over the years for the Schwarz algorithm. The first step is to introduce a symmetric variant of the ORAS (Optimized Restricted Additive Schwarz) algorithm 37 that is suitable for the analysis of a two-level method. Then we build a coarse space for which the convergence rate of the two-level method is guaranteed regardless of the regularity of the coefficients. We show scalability results for thousands of cores for nearly incompressible elasticity and the Stokes systems with a continuous discretization of the pressure.
\end{abstract}

\section{Introduction}

Substructuring algorithms such as BNN or FETI are defined for nonoverlapping domain decompositions but not for overlapping subdomains. Schwarz method [34] is defined only for overlapping subdomains. With the help of a coarse space correction, the two-level versions of both type of methods are weakly scalable, see 38 and references therein. The domain decomposition method introduced by P.L. Lions [27] is a third type of methods. It can be applied to both

*ryadh.haferssas@ljll.math.upmc.fr

†pierre.jolivet@enseeiht.fr

¥nataf@ann.jussieu.fr 
overlapping and nonoverlapping subdomains. It is based on improving Schwarz methods by replacing the Dirichlet interface conditions by Robin interface conditions. This algorithm was extended to Helmholtz problem by Després [9]. Robin interface conditions can be replaced by more general interface conditions that can be optimized (Optimized Schwarz methods, OSM) for a better convergence, see [18, 17] and references therein.

P.L. Lions proved the convergence of his algorithm in the elliptic case for a nonoverlapping domain decomposition. The proof is based on energy estimates and a summation technique. These results were extended to Helmholtz and Maxwell equations in [2, 10. Over the last years, a lot of results have been obtained for different classes of equations and optimized algorithms based on carefully chosen parameters in the transmission conditions, have been derived, see e.g. [21, 17, 18, 11] and references therein. Most of these works are valid for nonoverlapping decomposition or for simple overlapping domain decompositions. When the domain is decomposed into a large number of subdomains, these methods are, on a practical point of view, scalable if a second level is added to the algorithm via the introduction of a coarse space [21, 15, 17. But there is no systematic procedure to build coarse spaces with a provable efficiency.

The purpose of this article is to define a general framework for building adaptive coarse space for OSM methods for decomposition into overlapping subdomains. We prove that we can achieve the same robustness that what was done for Schwarz [35] and FETI-BDD [36] domain decomposition methods with so called GenEO (Generalized Eigenvalue in the Overlap) coarse spaces. Compared to these previous works, we have to introduce SORAS (symmetrized ORAS) a non standard symmetric variant of the ORAS method as well as two generalized eigenvalue problems. As numerical results will show in $\S 6.3$, the method scales very well for saddle point problems such as highly heterogeneous nearly incompressible elasticity problems as well as the Stokes system. More precisely, in $\S 2$, we give a short presentation of the current theory for the additive Schwarz method. Then, in section 3. we present algebraic variants to the P.L. Lions' domain decomposition method. In $\S 4$, we build a coarse space so that the twolevel SORAS method achieves a targeted condition number. In $\S 5$, the method is applied to saddle point problems.

\section{Short introduction to ASM theory}

In order to appraise the theory developed in $\S 3$, we first give a short presentation of the current theory for two-level additive Schwarz methods. The starting point was the original Schwarz algorithm 34 for proving the well-posedness of the Poisson problem $-\Delta u=f$ with Dirichlet boundary conditions in some domain $\Omega$ decomposed into two subdomains $\Omega_{1}$ and $\Omega_{2}, \Omega=\Omega_{1} \cup \Omega_{2}$.

Definition 2.1 (Original Schwarz algorithm) The Schwarz algorithm is an iterative method based on solving alternatively sub-problems in domains $\Omega_{1}$ and 


$$
\begin{aligned}
& \Omega_{2} \text {. It updates }\left(u_{1}^{n}, u_{2}^{n}\right) \rightarrow\left(u_{1}^{n+1}, u_{2}^{n+1}\right) \text { by : } \\
& \begin{array}{rllrlrl}
-\Delta\left(u_{1}^{n+1}\right) & =f & \text { in } \Omega_{1} & -\Delta\left(u_{2}^{n+1}\right) & =f & \text { in } \Omega_{2} \\
u_{1}^{n+1} & =0 & \text { on } \partial \Omega_{1} \cap \partial \Omega & \text { then, } & u_{2}^{n+1} & =0 & \text { on } \partial \Omega_{2} \cap \partial \Omega \\
u_{1}^{n+1} & =u_{2}^{n} & \text { on } \partial \Omega_{1} \cap \overline{\Omega_{2}} . & u_{2}^{n+1} & =u_{1}^{n+1} & \text { on } \partial \Omega_{2} \cap \overline{\Omega_{1}} .
\end{array}
\end{aligned}
$$

H. Schwarz proved the convergence of the algorithm and thus the well-posedness of the Poisson problem in complex geometries. A small modification of the algorithm [28] makes it suited to parallel architectures. Its convergence can be proved using the maximum principle [26].

Definition 2.2 (Parallel Schwarz algorithm) Iterative method which solves concurrently in all subdomains, $i=1,2$ :

$$
\begin{aligned}
& \begin{array}{rlll}
-\Delta\left(u_{i}^{n+1}\right) & =f & \text { in } & \Omega_{i} \\
u_{i}^{n+1} & =0 & \text { on } & \partial \Omega_{i} \cap \partial \Omega
\end{array} \\
& u_{i}^{n+1}=u_{3-i}^{n} \quad \text { on } \quad \partial \Omega_{i} \cap \bar{\Omega}_{3-i} .
\end{aligned}
$$

The discretization of this algorithm yields a parallel algebraic method for solving the linear system $A \mathbf{U}=\mathbf{F} \in \mathbb{R}^{\# \mathcal{N}}(\mathcal{N}$ is the set of degrees of freedom) arising from the discretization of the original Poisson problem set on domain $\Omega$. Due to the duplication of the unknowns in the overlapping region $\Omega_{1} \cap \Omega_{2}$, this direct discretization involves a matrix of size larger than that of matrix $A$, see e.g. 19] for more details. Actually, it is much simpler and as efficient to use the RAS preconditioner [6]

$$
M_{R A S}^{-1}:=\sum_{i=1}^{N} R_{i}^{T} D_{i} A_{i}^{-1} R_{i},
$$

where $N$ is the number of subdomains, $R_{i}$ for some $1 \leq i \leq N$ is the Boolean matrix that restricts a global vector to its degrees of freedom in subdomain $\Omega_{i}$, matrix

$$
A_{i}:=R_{i} A_{i} R_{i}^{T}
$$

is the Dirichlet matrix of subdomain $\Omega_{i}$ and $D_{i}$ is a local diagonal matrix that yields an algebraic partition of unity on $\mathbb{R}^{\# \mathcal{N}}$ :

$$
I_{d}=\sum_{i=1}^{N} R_{i}^{T} D_{i} R_{i}
$$

Indeed, it is proved in [14 that the following fixed point algorithm

$$
\mathbf{U}^{n+1}=\mathbf{U}^{n}+M_{R A S}^{-1}\left(\mathbf{F}-A \mathbf{U}^{n}\right)
$$

yields iterates that are equivalent to that of the discretization of Algorithm (2).

The RAS preconditioner (3) is not symmetric. In order to develop a theory for it when used as preconditioner in a Krylov method, its symmetric variant, 
the ASM preconditioner :

$$
M_{A S M}^{-1}=\sum_{i=1}^{N} R_{i}^{T} A_{i}^{-1} R_{i},
$$

was studied extensively, see 38 and references therein. Starting with the pioneering work 33, a lot of effort has been devoted to the design and analysis of two-level methods that are the key ingredient to scalable methods. In adaptive methods, the coarse space in the two-level method is built by solving local generalized eigenvalue problems [16, 13, 31, 35] . This way, it is possible to target a user defined condition number of the preconditioned system. Here we focus on the GenEO approach [35] where the coarse space is based on solving Generalized Eigenvalue problems for the set of degrees of freedom $\mathcal{N}_{j}$ of subdomain $1 \leq j \leq N$. Let $A_{j}^{N e u}$ denote the matrix of the local Neumann problem, we have to find the eigenpairs $\left(V_{j, k}, \lambda_{j, k}\right)_{k}$ such that:

$V_{j, k} \in \mathbb{R}^{\mathcal{N}_{j}}$ and $\lambda_{j, k} \geq 0$ :

$$
D_{j} A_{j} D_{j} V_{j, k}=\lambda_{j, k} A_{j}^{N e u} V_{j, k}
$$

By combining the eigenvectors corresponding to eigenvalues larger than some given threshold $\tau>0$ into a coarse space, it is proved in [35, 12] that the eigenvalues of the hybrid Schwarz preconditioned system satisfy the following estimate

$$
\frac{1}{1+k_{1} \tau} \leq \lambda\left(M_{H S M}^{-1} A\right) \leq k_{0} .
$$

where $k_{0}$ is the maximum number of neighbors of a subdomain and $k_{1}$ is the maximum multiplicity of the intersections of subdomains.

To sum up, the current theory for the two-level Schwarz method is based on the following four steps :

1. Schwarz algorithm at the continuous level (1)

2. An equivalent algebraic formulation (5) with the introduction of the RAS preconditioner (3)

3. A symmetrized variant named ASM (6) of the RAS preconditioner

4. A two-level method with an adaptive coarse space with prescribed targeted convergence rate.

\section{Symmetrized ORAS method}

Our goal here is to develop a theory and computational framework for P.L. Lions algorithm similar to what was done for the Schwarz algorithm for a SPD matrix $A$. We follow the steps recalled above. 
First we introduce the P.L. Lions' algorithm which is based on improving Schwarz methods by replacing the Dirichlet interface conditions by Robin interface conditions. Let $\alpha$ be a positive number, the modified algorithm reads

$$
\begin{aligned}
& -\Delta\left(u_{1}^{n+1}\right)=f \quad \text { in } \Omega_{1}, \\
& u_{1}^{n+1}=0 \\
& \left(\frac{\partial}{\partial \mathbf{n}_{1}}+\alpha\right)\left(u_{1}^{n+1}\right)=\left(\frac{\partial}{\partial \mathbf{n}_{1}}+\alpha\right)\left(u_{2}^{n}\right) \quad \text { on } \quad \partial \Omega_{1} \cap \overline{\Omega_{2}} \text {, }
\end{aligned}
$$

and

$$
\begin{aligned}
& -\Delta\left(u_{2}^{n+1}\right)=f \quad \text { in } \Omega_{2}, \\
& u_{2}^{n+1}=0 \\
& \text { on } \quad \partial \Omega_{2} \cap \partial \Omega \\
& \left(\frac{\partial}{\partial \mathbf{n}_{2}}+\alpha\right)\left(u_{2}^{n+1}\right)=\left(\frac{\partial}{\partial \mathbf{n}_{2}}+\alpha\right)\left(u_{1}^{n}\right) \quad \text { on } \quad \partial \Omega_{2} \cap \overline{\Omega_{1}}
\end{aligned}
$$

where $\mathbf{n}_{1}$ and $\mathbf{n}_{2}$ are the outward normals on the boundary of the subdomains.

The second step is an algebraic equivalent formulation of the P.L. Lions algorithm in the case of overlapping subdomains. It is based on the introduction of the ORAS (Optimized Restricted Additive Schwarz) [37] preconditioner :

$$
M_{\text {ORAS }}^{-1}:=\sum_{i=1}^{N} R_{i}^{T} D_{i} B_{i}^{-1} R_{i},
$$

where $\left(B_{i}\right)_{1 \leq i \leq N}$ is the discretization matrix of the Robin problem in subdomain $\Omega_{i}$. The following fixed point method

$$
\mathbf{U}^{n+1}=\mathbf{U}^{n}+M_{O R A S}^{-1}\left(\mathbf{F}-A \mathbf{U}^{n}\right)
$$

yields iterates that are equivalent to that of the discretization of P.L. Lions' Algorithm (9)-(10), see [37.

The third step is the introduction of a symmetric variant that allows for a comprehensive theoretical study. It seems at first glance that we should mimic what was done for the RAS algorithm and study the following symmetrized variant :

$$
M_{O A S, 1}^{-1}:=\sum_{i=1}^{N} R_{i}^{T} B_{i}^{-1} R_{i} .
$$

For reasons explained in Remark 1 we introduce another non standard variant of the ORAS preconditioner (11), the symmetrized ORAS (SORAS) algorithm :

$$
M_{S O R A S, 1}^{-1}:=\sum_{i=1}^{N} R_{i}^{T} D_{i} B_{i}^{-1} D_{i} R_{i} .
$$

The missing step is the fourth one, namely to build an adaptive coarse space for a two-level SORAS method. it is done in the next section. 


\section{Two-level SORAS algorithm}

Before designing and analyzing the two-level SORAS method, we precise our mathematical framework.

\subsection{Mathematical framework}

The problem to be solved is defined via a variational formulation on a domain $\Omega \subset \mathbb{R}^{d}$ for $d \in \mathbb{N}$ :

Find $u \in V$ such that $: a_{\Omega}(u, v)=l(v), \quad \forall v \in V$,

where $V$ is a Hilbert space of functions from $\Omega$ with real values, . The problem we consider is given through a symmetric positive definite bilinear form $a_{\Omega}$ that is defined in terms of an integral over any open set $\omega \subset \Omega$. Typical examples are the Darcy equation ( $\mathbf{K}$ is a diffusion tensor)

$$
a_{\omega}(u, v):=\int_{\omega} \mathbf{K} \nabla u \cdot \nabla v d x,
$$

or the elasticity system $(\boldsymbol{C}$ is the fourth-order stiffness tensor and $\boldsymbol{\varepsilon}(\boldsymbol{u})$ is the strain tensor of a displacement field $\boldsymbol{u})$ :

$$
a_{\omega}(\boldsymbol{u}, \boldsymbol{v}):=\int_{\omega} \boldsymbol{C}: \boldsymbol{\varepsilon}(\boldsymbol{u}): \varepsilon(\boldsymbol{v}) d x .
$$

The problem is discretized by a finite element method. Let $\mathcal{N}$ denote the set of degrees of freedom and $\left(\phi_{k}\right)_{k \in \mathcal{N}}$ be a finite element basis on a mesh $\mathcal{T}_{h}$. Let $A \in \mathbb{R}^{\# \mathcal{N} \times \# \mathcal{N}}$ be the associated finite element matrix, $A_{k l}:=a_{\Omega}\left(\phi_{l}, \phi_{k}\right)$, $k, l \in \mathcal{N}$. For some given right hand side $\mathbf{F} \in \mathbb{R}^{\# \mathcal{N}}$, we have to solve a linear system in $\mathbf{U}$ of the form

$$
A \mathbf{U}=\mathbf{F} .
$$

Domain $\Omega$ is decomposed into $N$ overlapping subdomains $\left(\Omega_{i}\right)_{1 \leq i \leq N}$ so that all subdomains are a union of cells of the mesh $\mathcal{T}_{h}$. This decomposition induces a natural decomposition of the set of indices $\mathcal{N}$ into $N$ subsets of indices $\left(\mathcal{N}_{i}\right)_{1 \leq i \leq N}$ :

$$
\mathcal{N}_{i}:=\left\{k \in \mathcal{N} \mid \text { meas }\left(\operatorname{supp}\left(\phi_{k}\right) \cap \Omega_{i}\right)>0\right\}, 1 \leq i \leq N .
$$

For all $1 \leq i \leq N$, let $R_{i}$ be the restriction matrix from $\mathbb{R}^{\# \mathcal{N}}$ to the subset $R^{\# \mathcal{N}_{i}}$ and $D_{i}$ be a diagonal matrix of size $\# \mathcal{N}_{i} \times \# \mathcal{N}_{i}$, so that we have a partition of unity at the algebraic level,

$$
\sum_{i=1}^{N} R_{i}^{T} D_{i} R_{i}=I_{d}
$$

where $I_{d} \in \mathbb{R}^{\# \mathcal{N} \times \# \mathcal{N}}$ is the identity matrix.

For all subdomains $1 \leq i \leq N$, let $B_{i}$ be a SPD matrix of size $\# \mathcal{N}_{i} \times \# \mathcal{N}_{i}$, 
which comes typically from the discretization of boundary value local problems using optimized transmission conditions.

We also define for all subdomains $1 \leq j \leq N, \widetilde{A}^{j}$, the $\# \mathcal{N}_{j} \times \# \mathcal{N}_{j}$ matrix defined by

$$
\mathbf{V}_{j}^{T} \widetilde{A}^{j} \mathbf{U}_{j}:=a_{\Omega_{j}}\left(\sum_{l \in \mathcal{N}_{j}^{j}} \mathbf{U}_{j l} \phi_{l}, \sum_{l \in \mathcal{N}_{j}} \mathbf{V}_{j l} \phi_{l}\right), \quad \mathbf{U}_{j}, \mathbf{V}_{j} \in \mathbb{R}^{\mathcal{N}_{j}} .
$$

When the bilinear form $a$ results from the variational solve of a Laplace problem, the previous matrix corresponds to the discretization of local Neumann boundary value problems. For this reason we will call it "Neumann" matrix even in a more general setting.

We also make use of two numbers $k_{0}$ and $k_{1}$ related to the domain decomposition. Let

$$
k_{0}:=\max _{1 \leq i \leq N} \#\left\{j \mid R_{j} A R_{i}^{T} \neq 0\right\}
$$

be the maximum multiplicity of the interaction between subdomains plus one. Let $k_{1}$ be the maximal multiplicity of subdomains intersection, i.e. the largest integer $m$ such that there exists $m$ different subdomains whose intersection has a non zero measure.

\subsection{SORAS with GenEO-2}

We now consider a two-level method based on enriching the one-level SORAS preconditioner (11) by introducing two generalized eigenvalue problems which allow us to control the spectrum of the preconditioned operator as written in Theorem 4.10,

\subsubsection{Coarse Space for the lower bound}

More precisely, we define the following generalized eigenvalue problem :

Definition 4.1 (Generalized Eigenvalue Problem for the lower bound) For each subdomain $1 \leq j \leq N$, we introduce the generalized eigenvalue problem

$$
\begin{gathered}
\text { Find }\left(\mathbf{V}_{j k}, \lambda_{j k}\right) \in \mathbb{R}^{\# \mathcal{N}_{j}} \backslash\{0\} \times \mathbb{R} \text { such that } \\
\widetilde{A}^{j} \mathbf{V}_{j k}=\lambda_{j k} B_{j} \mathbf{V}_{j k} .
\end{gathered}
$$

Let $\tau>0$ be a user-defined threshold, we define $Z_{\text {geneo }}^{\tau} \subset \mathbb{R}^{\# \mathcal{N}}$ as the vector space spanned by the family of vectors $\left(R_{j}^{T} D_{j} \mathbf{V}_{j k}\right)_{\lambda_{j k}<\tau, 1 \leq j \leq N}$ corresponding to eigenvalues smaller than $\tau$.

Let $\tilde{\pi}_{j}$ be the projection from $\mathbb{R}^{\# \mathcal{N}_{j}}$ on $\operatorname{Span}\left\{\mathbf{V}_{j k} \mid \lambda_{j k}<\tau\right\}$ parallel to $\operatorname{Span}\left\{\mathbf{V}_{j k} \mid \lambda_{j k} \geq\right.$ $\tau\}$. In the present case of the SORAS-2 method, Lemma 7.6, page 167 in [12] translates into :

Lemma 4.2 (Intermediate Lemma for GenEO-SORAS-2) For all subdomains $1 \leq j \leq N$ and $\mathbf{U}_{j} \in \mathbb{R}^{\mathcal{N}_{j}}$, we have :

$$
\tau\left(\left(I_{d}-\tilde{\pi}_{j}\right) \mathbf{U}_{j}\right)^{T} B_{j}\left(I_{d}-\tilde{\pi}_{j}\right) \mathbf{U}_{j} \leq \mathbf{U}_{j}^{T} \widetilde{A}^{j} \mathbf{U}_{j} .
$$




\subsubsection{Coarse space for the upper bound}

We introduce the following generalized eigenvalue problem :

Definition 4.3 (Generalized Eigenvalue Problem for the upper bound)

$$
\begin{gathered}
\text { Find }\left(\mathbf{U}_{i k}, \mu_{i k}\right) \in \mathbb{R}^{\# \mathcal{N}_{i}} \backslash\{0\} \times \mathbb{R} \text { such that } \\
\qquad D_{i} R_{i} A R_{i}^{T} D_{i} \mathbf{U}_{i k}=\mu_{i k} B_{i} \mathbf{U}_{i k} .
\end{gathered}
$$

Let $\gamma>0$ be a user-defined threshold, we define $Z_{\text {geneo }}^{\gamma} \subset \mathbb{R}^{\# \mathcal{N}}$ as the vector space spanned by the family of vectors $\left(R_{i}^{T} D_{i} \mathbf{U}_{i k}\right)_{\mu_{i k}>\gamma, 1 \leq i \leq N}$ corresponding to eigenvalues larger than $\gamma$.

Now, let $\xi_{i}$ denote the projection from $\mathbb{R}^{\mathcal{N}_{i}}$ on $\operatorname{Span}\left\{\mathbf{U}_{i k} \mid \gamma>\mu_{i k}\right\}$ parallel to $\operatorname{Span}\left\{\mathbf{U}_{i k} \mid \gamma \leq \mu_{i k}\right\}$. From these definitions, Lemma 7.6, page 167 in [12] leads to :

Lemma 4.4 For all subdomain $1 \leq i \leq N$ and $\mathbf{U}_{i} \in \mathbb{R}^{\# \mathcal{N}_{i}}$, we have :

$$
\left.\left(R_{i}^{T} D_{i}\left(I_{d}-\xi_{i}\right) \mathbf{U}_{i}\right)^{T} A R_{i}^{T} D_{i}\left(I_{d}-\xi_{i}\right) \mathbf{U}_{i}\right) \leq \gamma \mathbf{U}_{i}^{T} B_{i} \mathbf{U}_{i} .
$$

\subsection{SORAS-GENEO-2 method}

We are now ready to define the SORAS two level preconditioner

Definition 4.5 (Two level SORAS-GENEO-2 preconditioner) Let $P_{0}$ denote the a-orthogonal projection on the SORAS-GENEO-2 coarse space

$$
Z_{\text {GenEO-2 }}:=Z_{\text {geneo }}^{\tau} \bigoplus Z_{\text {geneo }}^{\gamma},
$$

the two-level SORAS-GENEO-2 preconditioner is defined as follows, see [29]:

$$
M_{S O R A S, 2}^{-1}:=P_{0} A^{-1}+\left(I_{d}-P_{0}\right) \sum_{i=1}^{N} R_{i}^{T} D_{i} B_{i}^{-1} D_{i} R_{i}\left(I_{d}-P_{0}^{T}\right) .
$$

Let $Z_{0}$ be a matrix whose columns are a basis of $Z_{\mathrm{GenEO}-2}$ and let denote its transpose by $R_{0}:=Z_{0}^{T}$. It is easily checked that

$$
P_{0} A^{-1}=R_{0}^{T}\left(R_{0} A R_{0}^{T}\right)^{-1} R_{0} .
$$

This definition is reminiscent of the balancing domain decomposition preconditioner [29] introduced for Schur complement based methods. Note that the coarse space is now defined by two generalized eigenvalue problems instead of one in 35, 36 for ASM and FETI-BDD methods.

The proof of Theorem 4.10 is based on the Fictitious Space [32] Lemma 7.4 in [12], page 164 . 
Definition 4.6 (Two-level SORAS in the Fictitious Space Lemma) Two Hilbert spaces $H$ and $H_{D}$, two other associated bilinear forms and induced scalar products as well as the $\mathcal{R}_{S O R A S, 2}$ operator between them are defined as follows.

- Space $H:=\mathbb{R}^{\# \mathcal{N}}$ endowed with the standard Euclidian scalar product. We consider another bilinear form a defined by:

$$
a: H \times H \rightarrow \mathbb{R}, \quad(\mathbf{U}, \mathbf{V}) \longmapsto a(\mathbf{U}, \mathbf{V}):=\mathbf{V}^{T} A \mathbf{U} .
$$

where $A$ is the matrix of the problem we want to solve.

- Space $H_{D}$, defined as the product space

$$
H_{D}:=\mathbb{R}^{\# \mathcal{N}_{0}} \times \prod_{i=1}^{N} \mathbb{R}^{\# \mathcal{N}_{i}}
$$

is endowed with standard scalar Euclidian product. For $\mathcal{U}=\left(\mathbf{U}_{i}\right)_{1 \leq i \leq N}$, $\mathcal{V}=\left(\mathbf{V}_{i}\right)_{1 \leq i \leq N}$ with $\mathbf{U}_{i}, \mathbf{V}_{i} \in \mathbb{R}^{\# \mathcal{N}_{i}}$, the bilinear form $b$ is defined by

$$
\begin{aligned}
b: H_{D} \times H_{D} & \longrightarrow \mathbb{R} \\
(\mathcal{U}, \mathcal{V}) & \longmapsto b(\mathcal{U}, \mathcal{V}):=\left(R_{0}^{T} \mathbf{V}_{0}\right)^{T} A\left(R_{0}^{T} \mathbf{U}_{0}\right)+\sum_{i=1}^{N} \mathbf{V}_{i}^{T} B_{i} \mathbf{U}_{i}
\end{aligned}
$$

Let $B$ denote the block-diagonal operator such that for all $\mathcal{U}, \mathcal{V} \in H_{D}$, we have:

$$
(B \mathcal{U}, \mathcal{V})=b(\mathcal{U}, \mathcal{V})
$$

- For any $\mathcal{U}=\left(\mathbf{U}_{i}\right)_{0 \leq i \leq N}$ the linear operator $\mathcal{R}_{S O R A S, 2}$ is defined as

$$
\mathcal{R}_{S O R A S, 2}: H_{D} \longrightarrow H, \mathcal{R}_{S O R A S, 2}(\mathcal{U}):=R_{0}^{T} \mathbf{U}_{0}+\sum_{i=1}^{N}\left(I_{d}-P_{0}\right) R_{i}^{T} D_{i} \mathbf{U}_{i} .
$$

It can easily be checked that

$$
M_{\text {SORAS }, 2}^{-1}=\mathcal{R}_{\text {SORAS }, 2} B^{-1} \mathcal{R}_{\text {SORAS }, 2}^{*} .
$$

We now check the assumptions of the Fictitious Space Lemma.

Lemma 4.7 (Surjectivity of $\mathcal{R}_{S O R A S, 2}$ ) Operator $\mathcal{R}_{S O R A S, 2}$ is surjective.

Proof

For all $\mathbf{U} \in H$, we have :

$$
\mathbf{U}=P_{0} \mathbf{U}+\left(I_{d}-P_{0}\right) \mathbf{U}=P_{0} \mathbf{U}+\sum_{i=1}^{N}\left(I_{d}-P_{0}\right) R_{i}^{T} D_{i} R_{i} \mathbf{U} .
$$

Since $P_{0} \mathbf{U} \in \operatorname{Span}\left(R_{0}^{T}\right)$, there exists $\mathbf{U}_{0} \in R^{\# \mathcal{N}_{0}}$ such that $P_{0} \mathbf{U}=R_{0}^{T} \mathbf{U}_{0}$. Thus, we have

$$
\mathbf{U}=R_{0}^{T} \mathbf{U}_{0}+\sum_{i=1}^{N}\left(I_{d}-P_{0}\right) R_{i}^{T} D_{i}\left(R_{i} \mathbf{U}\right),
$$


or, in other words

$$
\mathcal{R}_{S O R A S, 2}\left(\mathbf{U}_{0},\left(R_{i} \mathbf{U}\right)_{1 \leq i \leq N}\right)=\mathbf{U},
$$

which proves the surjectivity.

We now prove

Lemma 4.8 (Continuity of $\mathcal{R}_{S O R A S, 2}$ ) Let $\mathcal{U}=\left(\mathbf{U}_{i}\right)_{0 \leq i \leq N} \in H_{D}$. We have the following continuity estimate

$$
a\left(\mathcal{R}_{\text {SORAS,2}}(\mathcal{U}), \mathcal{R}_{\text {SORAS,2}}(\mathcal{U})\right) \leq \max \left(1, k_{0} \gamma\right) b(\mathcal{U}, \mathcal{U}) .
$$

Proof Since $P_{0}$ and $I_{d}-P_{0}$ are $a$-orthogonal projections, we have by $a$ orthogonality :

$$
\begin{aligned}
a\left(\mathcal{R}_{\text {SORAS }, 2}(\mathcal{U}), \mathcal{R}_{\text {SORAS }, 2}(\mathcal{U})\right)= & a\left(P_{0} R_{0}^{T} \mathbf{U}_{0}, P_{0} R_{0}^{T} \mathbf{U}_{0}\right) \\
& +a\left(\left(I_{d}-P_{0}\right) \sum_{i=1}^{N} R_{i}^{T} D_{i} \mathbf{U}_{i},\left(I_{d}-P_{0}\right) \sum_{i=1}^{N} R_{i}^{T} D_{i} \mathbf{U}_{i}\right)
\end{aligned}
$$

Since $P_{0}$ is the $a$-orthogonal projection on $Z_{\mathrm{GenEO}-2}$ and that

$$
\sum_{i=1}^{N} R_{i}^{T} D_{i} \xi_{i} \mathbf{U}_{i} \in Z_{\text {geneo }}^{\gamma} \subset Z_{\mathrm{GenEO}-2},
$$

we have

$$
\left(I_{d}-P_{0}\right) \sum_{i=1}^{N} R_{i}^{T} D_{i} \xi_{i} \mathbf{U}_{i}=0,
$$

and thus

$$
\begin{aligned}
& a\left(\left(I_{d}-P_{0}\right) \sum_{i=1}^{N} R_{i}^{T} D_{i} \mathbf{U}_{i},\left(I_{d}-P_{0}\right) \sum_{i=1}^{N} R_{i}^{T} D_{i} \mathbf{U}_{i}\right) \\
& \quad=a\left(\left(I_{d}-P_{0}\right) \sum_{i=1}^{N} R_{i}^{T} D_{i}\left(I_{d}-\xi_{i}\right) \mathbf{U}_{i},\left(I_{d}-P_{0}\right) \sum_{i=1}^{N} R_{i}^{T} D_{i}\left(I_{d}-\xi_{i}\right) \mathbf{U}_{i}\right)
\end{aligned}
$$

Finally, using $k_{0}$ defined as in in Lemma 7.11, page 174 in [12], we have

$$
\begin{aligned}
a\left(\mathcal{R}_{S O R A S, 2}(\mathcal{U}), \mathcal{R}_{S O R A S, 2}(\mathcal{U})\right) \leq & a\left(R_{0}^{T} \mathbf{U}_{0}, R_{0}^{T} \mathbf{U}_{0}\right) \\
& +a\left(\sum_{i=1}^{N} R_{i}^{T} D_{i}\left(I_{d}-\xi_{i}\right) \mathbf{U}_{i}, \sum_{i=1}^{N} R_{i}^{T} D_{i}\left(I_{d}-\xi_{i}\right) \mathbf{U}_{i}\right) \\
\leq & a\left(R_{0}^{T} \mathbf{U}_{0}, R_{0}^{T} \mathbf{U}_{0}\right) \\
& +k_{0} \sum_{i=1}^{N} a\left(R_{i}^{T} D_{i}\left(I_{d}-\xi_{i}\right) \mathbf{U}_{i}, R_{i}^{T} D_{i}\left(I_{d}-\xi_{i}\right) \mathbf{U}_{i}\right) .
\end{aligned}
$$

Then, using estimate 22 , we have :

$$
\begin{aligned}
a\left(\mathcal{R}_{\text {SORAS }, 2}(\mathcal{U}), \mathcal{R}_{\text {SORAS }, 2}(\mathcal{U})\right) & \leq a\left(R_{0}^{T} \mathbf{U}_{0}, R_{0}^{T} \mathbf{U}_{0}\right)+k_{0} \gamma \sum_{i=1}^{N}\left(B_{i} \mathbf{U}_{i}, \mathbf{U}_{i}\right) \\
& \leq \max \left(1, k_{0} \gamma\right) b(\mathcal{U}, \mathcal{U})
\end{aligned}
$$


which concludes the estimate of the continuity of $\mathcal{R}_{S O R A S, 2}$.

Lemma 4.9 (Stable decomposition with $\mathcal{R}_{S O R A S, 2}$ ) Let $\mathbf{U}$ be a vector in H. We define:

$$
\mathbf{U}_{j}:=\left(I_{d}-\tilde{\pi}_{j}\right) R_{j} \mathbf{U}
$$

and $\mathbf{U}_{0} \in \mathbb{R}^{\# \mathcal{N}_{0}}$ such that :

$$
R_{0}^{T} \mathbf{U}_{0}=P_{0} \mathbf{U}
$$

We define $\mathcal{U}:=\left(\mathbf{U}_{i}\right)_{0 \leq i \leq N}$.

Then, the stable decomposition property is verified with a constant $\left(1+k_{1} \tau^{-1}\right)^{-1}$, since we have :

$$
\begin{aligned}
\mathcal{R}_{\text {SORAS }, 2}(\mathcal{U}) & =\mathbf{U} \\
\frac{1}{\left(1+k_{1} \tau^{-1}\right)} b(\mathcal{U}, \mathcal{U}) & \leq a(\mathbf{U}, \mathbf{U}) .
\end{aligned}
$$

Proof We first check that we have indeed a decomposition $\mathcal{R}_{S O R A S, 2}(\mathcal{U})=\mathbf{U}$. Note that for all $1 \leq j \leq N$ we have

$$
R_{j}^{T} D_{j} \tilde{\pi}_{j} R_{j} \mathbf{U} \in Z_{\text {geneo }}^{\tau} \subset Z_{\mathrm{GenEO}-2} \Rightarrow\left(I_{d}-P_{0}\right) R_{j}^{T} D_{j} \widetilde{\pi}_{j} R_{j} \mathbf{U}=0 .
$$

We have :

$$
\begin{aligned}
\mathbf{U} & =P_{0} \mathbf{U}+\left(I_{d}-P_{0}\right) \mathbf{U}=P_{0} \mathbf{U}+\left(I_{d}-P_{0}\right) \sum_{j=1}^{N} R_{j}^{T} D_{j} R_{j} \mathbf{U} \\
& =P_{0} R_{0}^{T} \mathbf{U}_{0}+\left(I_{d}-P_{0}\right) \sum_{j=1}^{N} R_{j}^{T} D_{j}\left(I_{d}-\widetilde{\pi}_{j}\right) R_{j} \mathbf{U}=\mathcal{R}_{S O R A S, 2}(\mathcal{U}) .
\end{aligned}
$$

The last thing to do is to check the stability of this decomposition. Using 20 and then Lemma 7.13, page 175 in [12] ., we have

$$
\begin{aligned}
b(\mathcal{U}, \mathcal{U})= & a\left(R_{0}^{T} \mathbf{U}_{0}, R_{0}^{T} \mathbf{U}_{0}\right) \\
& \left.+\sum_{j=1}^{N}\left(\left(I_{d}-\widetilde{\pi}_{j}\right) R_{j} \mathbf{U}\right)^{T} B_{j}\left(\left(I_{d}-\widetilde{\pi}_{j}\right) R_{j} \mathbf{U}\right)\right) \\
\leq & a\left(P_{0} \mathbf{U}, P_{0} \mathbf{U}\right)+\tau^{-1} \sum_{j=1}^{N}\left(\widetilde{R}_{j} \mathbf{U}\right)^{T} \widetilde{A}^{j}\left(R_{j} \mathbf{U}\right) \\
\leq & a(\mathbf{U}, \mathbf{U})+k_{1} \tau^{-1} a(\mathbf{U}, \mathbf{U}) \leq\left(1+k_{1} \tau^{-1}\right) a(\mathbf{U}, \mathbf{U}) .
\end{aligned}
$$

The assumptions of the Fictitious Space Lemma are verified and thus we have just proved the following 
Theorem 4.10 (Spectral estimate for the two level SORAS-GenEO-2) Let $\gamma$ be a chosen threshold in Definition 4.3, $\tau$ be a chosen threshold in Definition (4.1) of the GenEO-2 coarse space and the two-level SORAS-GenEO-2 preconditioner defined by (23). Then, the eigenvalues of the two-level SORASGenEO-2 preconditioned system satisfy the following estimate

$$
\frac{1}{1+\frac{k_{1}}{\tau}} \leq \lambda\left(M_{S O R A S, 2}^{-1} A\right) \leq \max \left(1, k_{0} \gamma\right)
$$

We have the

Remark 1 An analysis of a two-level version of the preconditioner $M_{O A S}^{-1}(13$ following the same path yields the following two generalized eigenvalue problems :

$$
\begin{gathered}
\text { Find }\left(\mathbf{U}_{j k}, \mu_{j k}\right) \in \mathbb{R}^{\# \mathcal{N}_{i}} \backslash\{0\} \times \mathbb{R} \text { such that } \\
A^{i} \mathbf{U}_{i k}=\mu_{i k} B_{i} \mathbf{U}_{i k},
\end{gathered}
$$

and

$$
\begin{aligned}
\text { Find }\left(\mathbf{V}_{j k}, \lambda_{j k}\right) & \in \mathbb{R}^{\# \mathcal{N}_{i}} \backslash\{0\} \times \mathbb{R} \text { such that } \\
\widetilde{A}^{i} \mathbf{V}_{i k} & =\lambda_{i k} D_{i} B_{i} D_{i} \mathbf{V}_{i k} .
\end{aligned}
$$

In the general case for $1 \leq i \leq N$, matrices $D_{i}$ may have zero entries for boundary degrees of freedom since they are related to a partition of unity. Moreover very often matrices $B_{i}$ and $A_{i}$ differ only by the interface conditions that is for entries corresponding to boundary degrees of freedom. Therefore, matrix $D_{i} B_{i} D_{i}$ on the right hand side of the last generalized eigenvalue problem is not impacted by the choice of the interface conditions of the one level optimized Schwarz method. This cannot lead to efficient adaptive coarse spaces.

\section{$5 \quad$ Saddle point problems}

Many applications in science and engineering require solving large linear algebraic systems in saddle point form; see [3] for an extensive survey. Although our theory does not apply in a straightforward manner to saddle point problems, we use it for these difficult problems for which it is not always possible to preserve both symmetry and positivity of the problem, see [25. Note that generalized eigenvalue problems (21) and (19) still make sense if $A$ is the matrix of a saddle point problem and local matrices $A_{i}, B_{i}$ and $\widetilde{A}_{i}, 1 \leq i \leq N$, are based on a partition of unity and on variational formulations.

We start by the global problem defined via variational formulation see for instance $\S 6.1$ for the systems of almost incompressible elasticity and of Stokes. As in $\S 4.1$, these formulations are written in terms of integrals of differential quantities (gradient, divergence, ...) over some domain $\Omega \subset \mathbb{R}^{d}$ for $d \in \mathbb{N}$ :

Find $(\boldsymbol{u}, p) \in V \times \Lambda$ such that :

$$
\begin{aligned}
a_{\Omega}(\boldsymbol{u}, \boldsymbol{v})+b_{\Omega}(\boldsymbol{v}, p) & =l_{1}(\boldsymbol{v}), \quad \forall \boldsymbol{v} \in V, \\
b_{\Omega}(\boldsymbol{u}, q)-c_{\Omega}(p, q) & =l_{2}(q), \quad \forall q \in \Lambda,
\end{aligned}
$$


where $V$ and $\Lambda$ are Hilbert spaces of functions from $\Omega$ with real values, $a_{\Omega}, b_{\Omega}$ and $c_{\Omega}$ are bilinear forms, $a_{\Omega}$ and $b_{\Omega}$ being symmetric. Discretization by a finite element method yields a saddle point system of the form :

$$
A:=\left[\begin{array}{cc}
H & B^{T} \\
B & -C
\end{array}\right]\left[\begin{array}{l}
\boldsymbol{u} \\
p
\end{array}\right]=\left[\begin{array}{l}
\boldsymbol{f} \\
g
\end{array}\right]
$$

where $H=H^{T}$ is positive definite, $C=C^{T}$ is positive semidefinite. The set of degrees of freedom is decomposed into subsets $\left(\mathcal{N}_{i}\right)_{1 \leq i \leq N}$. The matrices involved in the partition of unity (16) have a block diagonal form

$$
D_{i}:=\left[\begin{array}{cc}
D_{i}^{u} & 0 \\
0 & D_{i}^{p}
\end{array}\right] \text { and } R_{i}:=\left[\begin{array}{cc}
R_{i}^{u} & 0 \\
0 & R_{i}^{p}
\end{array}\right] .
$$

The local "Dirichlet" matrices have the following block form :

$$
A_{i}:=R_{i} A R_{i}^{T}=\left[\begin{array}{cc}
H_{i} & B_{i}^{T} \\
B_{i} & -C_{i}
\end{array}\right]
$$

where

$$
H_{i}:=R_{i}^{u} H R_{i}^{u T}, \quad C_{i}:=R_{i}^{p} C R_{i}^{p T} \text { and } B_{i}:=R_{i}^{p} B R_{i}^{u T} .
$$

The local "Neumann" problems arise from the variational formulation restricted the finite element space of a subdomain as in (17). We use the following block notation

$$
\widetilde{A}_{i}:=\left[\begin{array}{cc}
\widetilde{H}_{i} & \widetilde{B}_{i}^{T} \\
\widetilde{B}_{i} & -\widetilde{C}_{i}
\end{array}\right] .
$$

For each subdomain $1 \leq i \leq N$, the "Robin" matrix is

$$
B_{i}=\widetilde{A}_{i}+Z_{i}
$$

where $Z_{i}=Z_{i}^{T}$ is positive semidefinite and is such that matrix $B_{i}$ is symmetric positive definite. For sake of simplicity the "Robin" boundary condition will only apply to the $\boldsymbol{u}$ term, that is :

$$
Z_{i}=\left[\begin{array}{cc}
Z_{i}^{u} & 0 \\
0 & 0
\end{array}\right]
$$

\subsection{GenEO eigenvalue problem for saddle point problems}

Eigenvalue problem for saddle point problem has been considered by various authors, see [4] and references therein. We cannot use directly their results since we consider generalized eigenvalue problems where both left and right matrices have saddle point structures. In order to prove that the GenEO eigenvalues are real and non negative, we need the following assumption :

\section{Assumption 1}

$$
\left(\widetilde{H}_{i} \boldsymbol{u}, \boldsymbol{u}\right)+\left(Z_{i}^{u} \boldsymbol{u}, \boldsymbol{u}\right)+\left(\widetilde{C}_{i} p, p\right)=0 \Rightarrow \boldsymbol{u}=0 \text { and } p=0 .
$$


Consider the generalized eigenvalue problem that controls the lower bound of the spectrum of the preconditioned system :

$$
\left[\begin{array}{cc}
\widetilde{H}_{i} & \widetilde{B}_{i}^{T} \\
\widetilde{B}_{i} & -\widetilde{C}_{i}
\end{array}\right]\left[\begin{array}{l}
\boldsymbol{u} \\
p
\end{array}\right]=\lambda\left[\begin{array}{cc}
\widetilde{H}_{i}+Z_{i}^{u} & \widetilde{B}_{i}^{T} \\
\widetilde{B}_{i} & -\widetilde{C}_{i}
\end{array}\right]\left[\begin{array}{l}
\boldsymbol{u} \\
p
\end{array}\right] .
$$

We take the scalar product of 30 with $[\boldsymbol{u}-p]^{T}$. The cross product terms $\left(\widetilde{B}_{i}^{T} \boldsymbol{u}, p\right)$ cancel and we get :

$$
\left(\widetilde{H}_{i} \boldsymbol{u}, \boldsymbol{u}\right)+\left(\widetilde{C}_{i} p, p\right)=\lambda\left[\left(\widetilde{H}_{i} \boldsymbol{u}, \boldsymbol{u}\right)+\left(Z_{i}^{u} \boldsymbol{u}, \boldsymbol{u}\right)+\left(\widetilde{C}_{i} p, p\right)\right] .
$$

All terms above are non negative. From Assumption 1 the right term cannot be zero. Therefore, $\lambda \in[0,1]$.

Consider now the eigenvalue problem that controls the upper bound of the spectrum of the preconditioned system :

$$
\left[\begin{array}{cc}
D_{i}^{u} H_{i} D_{i}^{u} & D_{i}^{u} B_{i}^{T} D_{i}^{p} \\
D_{i}^{p} B_{i} D_{i}^{u} & -D_{i}^{p} C_{i} D_{i}^{p}
\end{array}\right]\left[\begin{array}{l}
\boldsymbol{u} \\
p
\end{array}\right]=\mu\left[\begin{array}{cc}
\widetilde{H}_{i}+Z_{i}^{u} & \widetilde{B}_{i}^{T} \\
\widetilde{B}_{i} & -\widetilde{C}_{i}
\end{array}\right]\left[\begin{array}{l}
\boldsymbol{u} \\
p
\end{array}\right]
$$

We take the scalar product of $\left[32\right.$ with $[\boldsymbol{u}-p]^{T}$ and we get :

$$
\left(H_{i} D_{i}^{u} \boldsymbol{u}, D_{i}^{u} \boldsymbol{u}\right)+\left(C_{i} D_{i}^{p} p, D_{i}^{p} p\right)=\mu\left[\left(\widetilde{H}_{i} \boldsymbol{u}, \boldsymbol{u}\right)+\left(Z_{i}^{u} \boldsymbol{u}, \boldsymbol{u}\right)+\left(\widetilde{C}_{i} p, p\right)\right]
$$

All terms above are non negative. From Assumption 1 , the right term cannot be zero. Therefore, $\mu \geq 0$.

\section{Application to the systems of Stokes and of Nearly Incompressible elasticity}

Mixed finite elements are often used to solve incompressible Stokes and nearly incompressible elasticity problems. Continuous pressures have been used in many mixed finite elements. However, most domain decomposition methods require that the pressure be discontinuous when they are used to solve the indefinite linear systems arising from such mixed finite element discretizations. Several domain decomposition algorithms allow one to use continuous pressures, see [39] and references therein. To our knowledge, our method is the first one to exhibit scalability for a highly heterogeneous nearly incompressible elasticity problems with continuous pressures.

\subsection{Variational formulations}

The mechanical properties of a solid can be characterized by its Young modulus $E$ and Poisson ratio $\nu$ or alternatively by its Lamé coefficients $\lambda$ and $\mu$. These coefficients relate to each other by the following formulas:

$$
\lambda=\frac{E \nu}{(1+\nu)(1-2 \nu)} \text { and } \mu=\frac{E}{2(1+\nu)} .
$$


The variational problem consists in finding $\left(\boldsymbol{u}_{h}, p_{h}\right) \in \mathcal{V}_{h}:=\mathbb{P}_{2}^{d} \cap H_{0}^{1}(\Omega) \times \mathbb{P}_{1}$ such that for all $\left(\boldsymbol{v}_{h}, q_{h}\right) \in \mathcal{V}_{h}$

$$
\begin{cases}\int_{\Omega} 2 \mu \underline{\underline{\varepsilon}}\left(\boldsymbol{u}_{h}\right): \underline{\underline{\varepsilon}}\left(\boldsymbol{v}_{h}\right) d x & -\int_{\Omega} p_{h} \operatorname{div}\left(\boldsymbol{v}_{h}\right) d x=\int_{\Omega} \boldsymbol{f} \boldsymbol{v}_{h} d x \\ -\int_{\Omega} \operatorname{div}\left(\boldsymbol{u}_{h}\right) q_{h} d x & -\int_{\Omega} \frac{1}{\lambda} p_{h} q_{h}=0\end{cases}
$$

Let $\boldsymbol{u}$ denote the degrees of freedom of $\boldsymbol{u}_{h}$ and $p$ that of $p_{h}$, they satisfy a linear system denoted as follows :

$$
A \mathbf{U}=\left[\begin{array}{cc}
H & B^{T} \\
B & -C
\end{array}\right]\left[\begin{array}{l}
\boldsymbol{u} \\
p
\end{array}\right]=\left[\begin{array}{l}
\mathrm{f} \\
0
\end{array}\right]=\mathbf{F} .
$$

Matrix $\widetilde{A}_{i}$ arises from the variational formulation 35 where the integration over domain $\Omega$ is replaced by the integration over subdomain $\Omega_{i}$ and finite element space $\mathcal{V}_{h}$ is restricted to subdomain $\Omega_{i}$. Matrix $B_{i}$ corresponds to a Robin problem and is the sum of matrix $\widetilde{A}_{i}$ and of the matrix of the following variational formulation restricted to the same finite element space :

$$
\int_{\partial \Omega_{i} \backslash \partial \Omega} \frac{2 \alpha \mu(2 \mu+\lambda)}{\lambda+3 \mu} \boldsymbol{u}_{h} \cdot \boldsymbol{v}_{h} \text { with } \alpha=10 \text { in our test. }
$$

In the next section, we explain the origin of the term 37 .

\subsection{Interface conditions}

We touch here another peculiarity of the P.L. Lions algorithm. In some situations, it is possible to choose the interface condition in order to have convergence in a number of steps equal to the number of subdomains, see 30. In our case, let the global domain $\Omega$ be the whole plane $\mathbb{R}^{2}$ decomposed into two half planes $\Omega_{1}:=(-\infty, \delta) \times \mathbb{R}$ and $\Omega_{2}:=(0, \infty) \times \mathbb{R}$ where $\delta \geq 0$ is the width of the overlap, $k$ denote the Fourier transform in the $y$ direction, the following interface condition yields to a convergence in two iterations :

$$
\underline{\underline{\boldsymbol{\sigma}}} \cdot \underline{\boldsymbol{n}}+\mathcal{F}^{-1}\left(\left[\begin{array}{cc}
\frac{2|k| \mu(2 \mu+\lambda)}{\lambda+3 \mu} & \frac{2 i k \mu^{2}}{\lambda+3 \mu} \\
\frac{-2 i k \mu^{2}}{\lambda+3 \mu} & \frac{2|k| \mu(2 \mu+\lambda)}{\lambda+3 \mu}
\end{array}\right]\left[\begin{array}{l}
\mathcal{F}\left(u_{x}\right) \\
\mathcal{F}\left(u_{y}\right)
\end{array}\right]\right)
$$

where $\underline{\underline{\boldsymbol{\sigma}}} \cdot \underline{\boldsymbol{n}}$ is the normal component of the stress tensor, the velocity is decomposed into its normal $u_{x}$ and tangential component $u_{y} \boldsymbol{u}=\left[u_{x}, u_{y}\right]^{T}$ and $\mathcal{F}$ denotes the Fourier transform in the $y$ direction. Due to the absolute value $|k|$ this interface condition is non local in space and also difficult to apply in the general domain decompositions we have here. For sake of simplicity, we choose

some frequency $k_{0}$ and we approximate the optimal interface condition (38) as follows :

$$
\underline{\underline{\boldsymbol{\sigma}}} \cdot \underline{\boldsymbol{n}}+\mathcal{F}^{-1}\left(\left[\begin{array}{cc}
\frac{2\left|k_{0}\right| \mu(2 \mu+\lambda)}{\lambda+3 \mu} & 0 \\
0 & \frac{2\left|k_{0}\right| \mu(2 \mu+\lambda)}{\lambda+3 \mu}
\end{array}\right]\left[\begin{array}{c}
\mathcal{F}\left(u_{x}\right) \\
\mathcal{F}\left(u_{y}\right)
\end{array}\right]\right)
$$




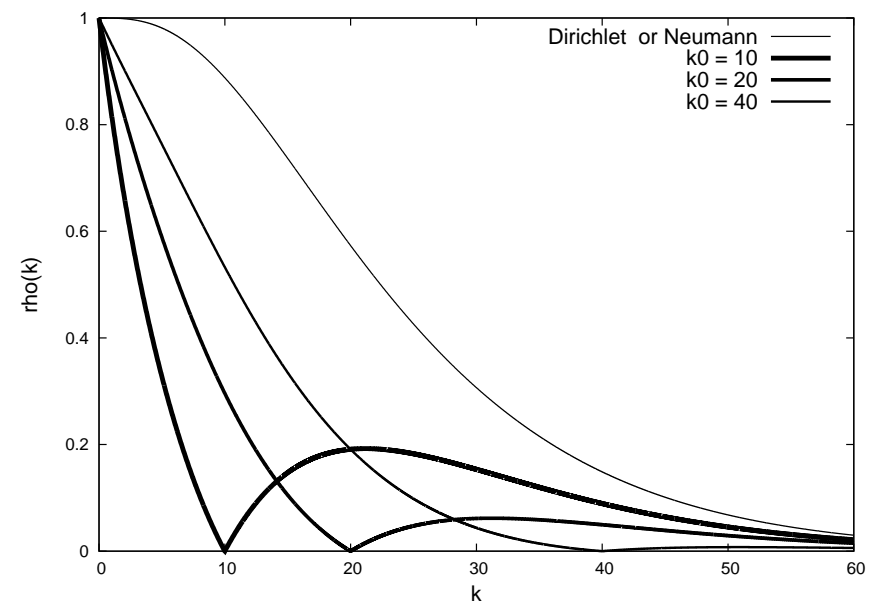

Figure 1 - Convergence rate vs Fourier number $k$ for various interface conditions - Poisson ratio $\nu=0.4999-$ overlap $\delta=0.1$.

which simplifies in :

$$
\underline{\underline{\boldsymbol{\sigma}}} \cdot \underline{\boldsymbol{n}}+\left|k_{0}\right| \frac{2 \mu(2 \mu+\lambda)}{\lambda+3 \mu} \boldsymbol{u} .
$$

This approximation has an impact on the convergence rate of the P.L. Lions' algorithm. On Figure 1, we plot the convergence as a function of the Fourier mode in the $y$ direction for various interface conditions for an almost incompressible elasticity system $\nu=0.4999$ and an overlap of size $\delta=0.1$. Although the Robin interface condition (39) is never exact even for $k=k_{0}$, the convergence rate is quite close to zero (of the order of $10^{-4}$ ) for $k=k_{0}$. Note that Dirichlet or Neumann (stress free) interface conditions yield the same convergence rates. For small Fourier numbers, the convergence rate is very close to 1 which is bad. Overall, Robin interface conditions perform much better than simple Dirichlet or Neumann interface conditions. The interface condition (39) can be used for arbitrary domain decompositions since its variational formulation is the one of a stress free BVP to which we add the variational formulation of (37) where $\alpha:=\left|k_{0}\right|$ for some chosen Fourier number $k_{0}$. Thus although the Fourier analysis has a limited domain of validity, the interface condition $(39)$ can be used for arbitrary domain decompositions.

\subsection{Numerical results}

The new coarse space was tested quite successfully on nearly incompressible elasticity and Stokes problems with a discretization based on saddle point 


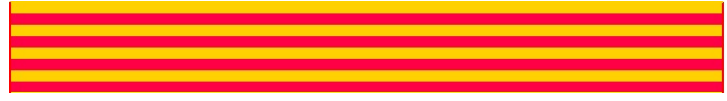

FiguRE 2-2D Elasticity : coefficient distribution of steel and rubber.

\begin{tabular}{|r|c||c||c||c|c||c|c||c|c||c|c|}
\hline & & AS & SORAS & AS+ZEM & \multicolumn{2}{|c||}{ SORAS +ZEM } & \multicolumn{2}{|c|}{ AS-GenEO } & \multicolumn{2}{|c|}{ SORAS GenEO2 } \\
\hline d.o.f. & $N$ & iter & iter & iter & dim & iter & dim & iter & dim & iter & dim \\
\hline 35841 & 8 & 150 & 184 & 117 & 24 & 79 & 24 & 110 & 184 & 13 & 145 \\
\hline 70590 & 16 & 276 & 337 & 170 & 48 & 144 & 48 & 153 & 400 & 17 & 303 \\
\hline 141375 & 32 & 497 & $>1000$ & 261 & 96 & 200 & 96 & 171 & 800 & 22 & 561 \\
\hline 279561 & 64 & $>1000$ & $>1000$ & 333 & 192 & 335 & 192 & 496 & 1600 & 24 & 855 \\
\hline 561531 & 128 & $>1000$ & $>1000$ & 329 & 384 & 400 & 384 & $>1000$ & 2304 & 29 & 1220 \\
\hline 1077141 & 256 & $>1000$ & $>1000$ & 369 & 768 & $>1000$ & 768 & $>1000$ & 3840 & 36 & 1971 \\
\hline
\end{tabular}

TABLE 1 - 2D Elasticity. GMRES iteration counts for a solid made of steel and rubber.

formulations in order to avoid locking phenomena.

\subsubsection{Tests against other algorithms}

We first report 2D results for a heterogeneous beam of eight layers of steel $\left(E_{1}, \nu_{1}\right)=\left(210 \cdot 10^{9}, 0.3\right)$ and rubber $\left(E_{2}, \nu_{2}\right)=\left(0.1 \cdot 10^{9}, 0.4999\right)$, see Figure 2 The beam is clamped on its left and right sides. Simulations were made with FreeFem ++ 20]. Iteration counts for various domain decomposition methods for a relative tolerance of $10^{-6}$ are given in Table 1 . We compare the one level Additive Schwarz (AS) and SORAS methods, the two level AS and SORAS methods with a coarse space consisting of rigid body motions which are zero energy modes (ZEM) and finally AS with a GenEO coarse space as defined in [35] and SORAS with the GenEO-2 coarse space defined in Definition 4.1 with $\tau=0.4$ and $\gamma=10^{3}$. Columns dim refer to the total size of the coarse space of a two-level method. Eigenvalue problem (19) accounts for roughly $90 \%$ of the GenEO-2 coarse space size. We see that only the last method scales well with respect to the number of subdomains denoted by $N$.

\subsubsection{D and 2D highly heterogeneous linear elasticity equations}

Throughout this section we look at a linear elasticity problem with highly heterogeneous Lamé coefficients corresponding to steel and rubber materials. In the case of rubber which is nearly incompressible material the Poisson ratio $\nu$ approaches $1 / 2$ and $\lambda / \mu=2 \nu /(1-2 \nu)$ approaches infinity. In order to avoid the resulting locking phenomena with finite element discretization, the pure displacement problem is replaced by a mixed formulation as proposed in [5]. We performed a large 2D and 3D simulations, on an heterogeneous beam, where the Lamé $(E, \nu)$ vary discontinuously over the domain in eight alternating layers of steel material with $\left(E_{1}, \nu_{1}\right)=\left(210 \times 10^{9}, 0.3\right)$ and rubber material with $\left(E_{2}, \nu_{2}\right)=\left(0.1 \times 10^{9}, 0.4999\right)$ submitted to an external forces, see Figure 3 . The system is discretized using a Taylor-Hood mixed finite element discretization 

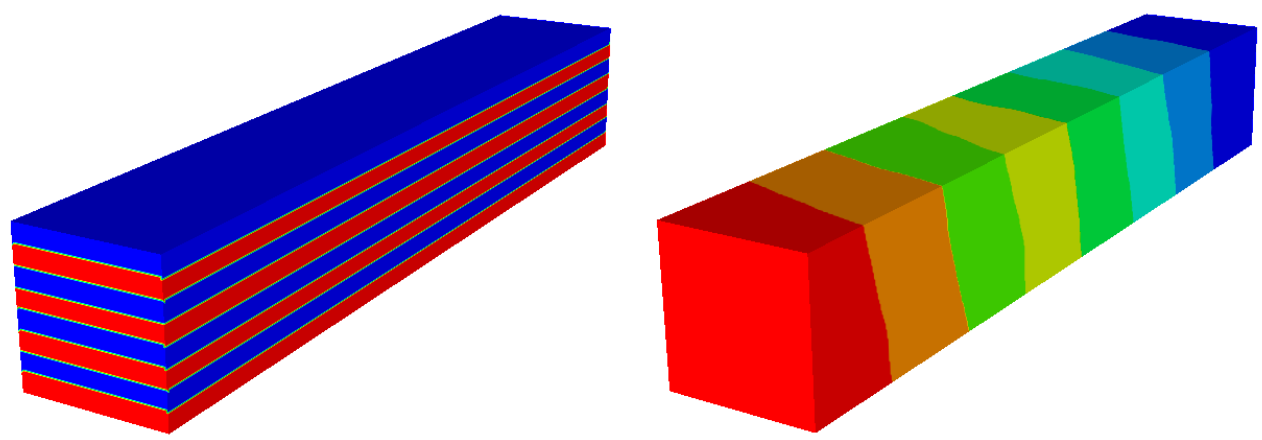

Figure 3 - Material coefficient, alternating layers of steel and rubber (left) and domain decomposition into 8 subdomains with a graph partitioner (right)

which are inf-sup stable. $P_{3} / P_{2}$ for the $2 \mathrm{D}$ case and $P_{2} / P_{1}$ for the $3 \mathrm{D}$ case.

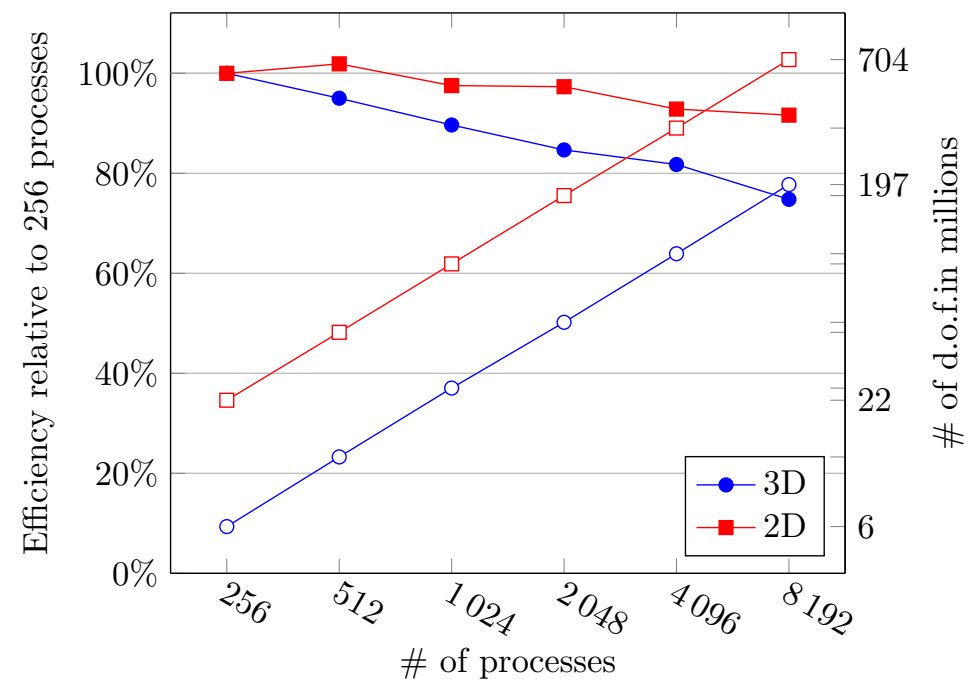

FiguRE 4 - Weak scaling experiments.

The problem is solved with a minimal geometric overlap of one mesh element and a preconditioned GMRES is used to solve the resulting linear system where the stopping criteria for the relative residual norm is fixed to $10^{-6}$. All the test cases were performed inside FreeFem ++ code interfaced with the domain decomposition library HPDDM [22, 23. The factorizations are computed for each local problem and also for the global coarse problem using MUMPS [1. Generalized eigenvalue problems to generate the GenEO space are solved using ARPACK 24]. The coarse space is formed only with the generalized eigenvalue problem (19) since we noticed that the second one (21) has only a little effect on 


\begin{tabular}{|c|c|c|c|c|c|c|c|}
\hline & $N$ & Factorization & Deflation & Solution & $\#$ of it. & Total & \# of d.o.f. \\
\hline \multirow{6}{*}{$3 \mathrm{D}$} & 256 & $25.2 \mathrm{~s}$ & $76.0 \mathrm{~s}$ & $37.2 \mathrm{~s}$ & 46 & $145.2 \mathrm{~s}$ & $6.1 \cdot 10^{6}$ \\
\hline & 512 & $26.5 \mathrm{~s}$ & $81.1 \mathrm{~s}$ & $39.8 \mathrm{~s}$ & 47 & $155.1 \mathrm{~s}$ & $12.4 \cdot 10^{6}$ \\
\hline & 1024 & $29.2 \mathrm{~s}$ & $82.6 \mathrm{~s}$ & $41.7 \mathrm{~s}$ & 45 & $165.5 \mathrm{~s}$ & $25.0 \cdot 10^{6}$ \\
\hline & 2048 & $26.9 \mathrm{~s}$ & $83.5 \mathrm{~s}$ & $46.3 \mathrm{~s}$ & 47 & $171.0 \mathrm{~s}$ & $48.8 \cdot 10^{6}$ \\
\hline & 4096 & $28.3 \mathrm{~s}$ & $88.8 \mathrm{~s}$ & $54.5 \mathrm{~s}$ & 53 & $177.7 \mathrm{~s}$ & $97.9 \cdot 10^{6}$ \\
\hline & 8192 & $29.0 \mathrm{~s}$ & $78.3 \mathrm{~s}$ & $79.8 \mathrm{~s}$ & 60 & $196.1 \mathrm{~s}$ & $197.6 \cdot 10^{6}$ \\
\hline \multirow{6}{*}{$2 \mathrm{D}$} & 256 & $4.8 \mathrm{~s}$ & $72.9 \mathrm{~s}$ & $39.9 \mathrm{~s}$ & 46 & $123.9 \mathrm{~s}$ & $22.1 \cdot 10^{6}$ \\
\hline & 512 & $4.7 \mathrm{~s}$ & $65.9 \mathrm{~s}$ & $45.0 \mathrm{~s}$ & 51 & $121.3 \mathrm{~s}$ & $44.0 \cdot 10^{6}$ \\
\hline & 1024 & $4.8 \mathrm{~s}$ & $70.0 \mathrm{~s}$ & $46.1 \mathrm{~s}$ & 51 & $127.0 \mathrm{~s}$ & $88.3 \cdot 10^{6}$ \\
\hline & 2048 & $4.8 \mathrm{~s}$ & $69.0 \mathrm{~s}$ & $46.5 \mathrm{~s}$ & 51 & $127.4 \mathrm{~s}$ & $176.8 \cdot 10^{6}$ \\
\hline & 4096 & $4.8 \mathrm{~s}$ & $65.8 \mathrm{~s}$ & $52.8 \mathrm{~s}$ & 56 & $132.6 \mathrm{~s}$ & $351.0 \cdot 10^{6}$ \\
\hline & 8192 & $4.8 \mathrm{~s}$ & $65.4 \mathrm{~s}$ & $53.0 \mathrm{~s}$ & 54 & $134.8 \mathrm{~s}$ & $704.1 \cdot 10^{6}$ \\
\hline
\end{tabular}

FIGURE 5 - Weak scaling experiments elasticity timings tab .

the convergence. All the results of this section were obtained on Turing machine which is an IBM/Blue Gene/Q machine composed of 1024 compute nodes where each one is made of 16 cores PowerPC A2 clocked at $1.6 \mathrm{GHz}$.

These computations, see Figure 4, assess the weak scalability of the algorithm with respect to the problem size and the number of subdomains. All times are wall clock times. The domain is decomposed automatically into subdomains with a graph partitioner, ranging from 256 subdomains to 8192 . and the problem size is increased by mesh refinement. In 3D the initial problem is about 6 millions d.o.f decomposed into 256 subdomains and solved in $145.2 \mathrm{~s}$ and the final problem is about 197 millions of d.o.f decomposed into 8192 subdomains and solved in $196 \mathrm{~s}$ which gives an efficiency near to $75 \%$. For the $2 \mathrm{D}$ case, the initial problem is approximatively of size 22 millions unknowns (d.o.f) decomposed into 256 subdomains and solved in 123.9s and we end up with a bigger problem about 704 millions unknowns (d.o.f) decomposed into 8192 subdomains and solved in 134s. The efficiency is close to $90 \%$. In figure table 5, we report the number of GMRES iterations. Thanks to the robustness of the two-level domain decomposition preconditioner we can observe that they are quite good stable. We report in the same table all the timings concerning the algorithm, column "Factorization" concerns the local subdomains, the assembling and the factorization of the coarse operator are in column "Deflation" and in column "Solution" we display the time spent by GMRES.

\subsection{D and 2D incompressible Stokes system}

Using the same libraries, we also performed a strong scaling test for an incompressible Stokes system of equations for a driven cavity problem : 
Find $(\boldsymbol{u}, \boldsymbol{p}) \in H(\Omega)^{d=2,3} \times L_{0}(\Omega)$ such that

$$
-\operatorname{div} \underline{\underline{\sigma}}_{F}(\boldsymbol{u}, \boldsymbol{p})=0, \quad \text { and } \operatorname{div}(\boldsymbol{u})=0 \quad \text { in } \Omega,
$$

with

$$
\left\{\begin{array}{l}
\underline{\underline{\sigma}_{F}}(\boldsymbol{u}, \boldsymbol{p})=-p I+2 \mu \underline{\underline{\varepsilon}}(\boldsymbol{u}), \\
\underline{\underline{\varepsilon}}(\boldsymbol{u})=\frac{1}{2}\left(\underline{\underline{\nabla}} \boldsymbol{u}+(\underline{\underline{\nabla}} \boldsymbol{u})^{T}\right) \text { and } \varepsilon_{i, j}=\frac{1}{2}\left(\frac{\partial u_{i}}{\partial x_{j}}+\frac{\partial u_{j}}{\partial x_{i}}\right) .
\end{array}\right.
$$

As a boundary conditions, we consider a continuous velocity on the upper face and zero Dirichlet otherwise. The computations are done in both two and three dimensions on a domain $\Omega=[0,1]^{2}$ and $\Omega=[0,1]^{3}$, respectively. Once more the problems are discretized via Taylor-Hood finite element $P_{2} / P_{1}$ with a continuous pressure.

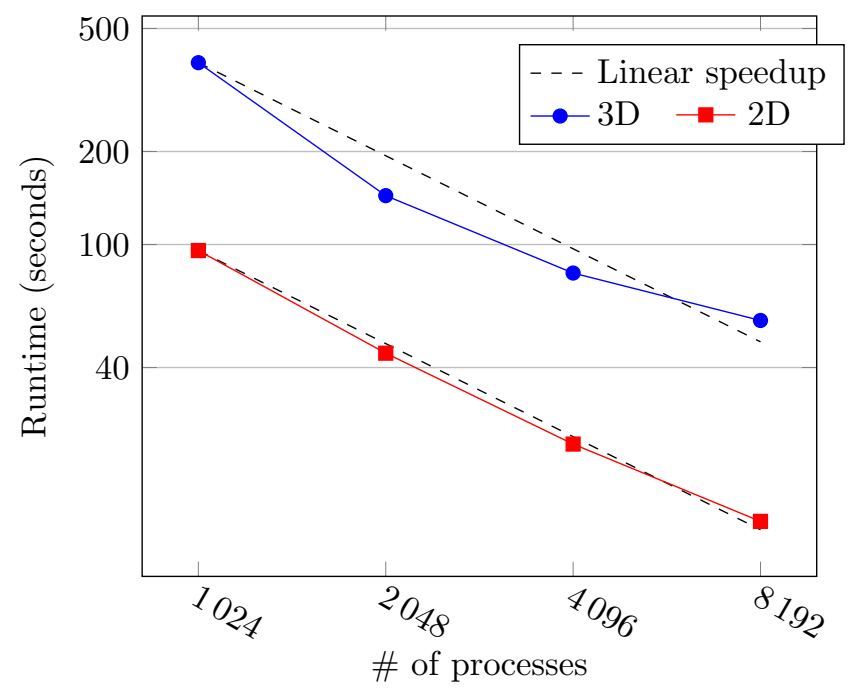

FiguRE 6 - Timings of various simulations Stokes.

We assess here the strong scalability of the algorithm. For this, we make the number of subdomains vary for a fixed global system size. In our test case the system size is fixed to 50 millions unknowns (d.o.f) in 3D and to 100 millions unknowns (d.o.f) in 2D, as we can show in figure 6, from 1024 subdomains to 8192 subdomains we get a quite good speed up. In the three dimensional case, we pass from 387.5s using 1024 subdomains to 56.8 s when using 8192 subdomains. In figure table 7 we display all timings relative to this test, column "Factorization" gives the time spent in the factorization of the local submatrices, column "Deflation" corresponds to local eigenvalue solvers and the coarse space correction construction, column "Solution" is the time taken by the GMRES solve of the global linear system by the domain decomposition algorithm. 


\begin{tabular}{rrrrrrrr}
\hline & $N$ & Factorization & Deflation & Solution & \# of it. & Total & \# of d.o.f. \\
\hline \multirow{3}{*}{$3 \mathrm{D}$} & 1024 & $79.2 \mathrm{~s}$ & $229.0 \mathrm{~s}$ & $76.3 \mathrm{~s}$ & 45 & $387.5 \mathrm{~s}$ & \\
& 2048 & $29.5 \mathrm{~s}$ & $76.5 \mathrm{~s}$ & $34.8 \mathrm{~s}$ & 42 & $143.9 \mathrm{~s}$ & $50.63 \cdot 10^{6}$ \\
& 4096 & $11.1 \mathrm{~s}$ & $45.8 \mathrm{~s}$ & $19.8 \mathrm{~s}$ & 42 & $80.9 \mathrm{~s}$ & \\
& 8192 & $4.7 \mathrm{~s}$ & $26.1 \mathrm{~s}$ & $14.9 \mathrm{~s}$ & 41 & $56.8 \mathrm{~s}$ & \\
\hline \multirow{3}{*}{$2 \mathrm{D}$} & 1024 & $5.2 \mathrm{~s}$ & $37.9 \mathrm{~s}$ & $51.5 \mathrm{~s}$ & 51 & $95.6 \mathrm{~s}$ & \\
& 2048 & $2.4 \mathrm{~s}$ & $19.3 \mathrm{~s}$ & $22.1 \mathrm{~s}$ & 42 & $44.5 \mathrm{~s}$ & $100.13 \cdot 10^{6}$ \\
& 4096 & $1.1 \mathrm{~s}$ & $10.4 \mathrm{~s}$ & $10.2 \mathrm{~s}$ & 35 & $22.6 \mathrm{~s}$ & \\
& 8192 & $0.5 \mathrm{~s}$ & $4.6 \mathrm{~s}$ & $6.9 \mathrm{~s}$ & 38 & $12.7 \mathrm{~s}$ & \\
\hline
\end{tabular}

FIGURE 7 - Strong scaling experiments Stokes.

\section{Conclusion}

We developed a theory for the overlapping P.L. Lions' algorithm similar to the existing one for the Schwarz algorithm in that we show how to build adaptively a coarse space so that the two-level preconditioner achieves a targeted condition number. The theory is based on the introduction of the SORAS (14) algorithm which is a new symmetric variant of the ORAS preconditioner. The two-level method is implemented in the HPDDM library that is interfaced with finite element solvers such as FreeFem ++ and Feel++.

Note that for a given targeted condition number, the size of the coarse space depends on the interface condition. A small coarse space is important in order to achieve good scalability results. Thus, it might be interesting to optimize this condition with respect to the coarse space size.

\section{Références}

[1] Patrick R. Amestoy, Iain S. Duff, Jean-Yves L'Excellent, and Jacko Koster. A fully asynchronous multifrontal solver using distributed dynamic scheduling. SIAM J. Matrix Analysis and Applications, 23(1) :15-41, 2001.

[2] Jean-David Benamou and Bruno Desprès. A domain decomposition method for the Helmholtz equation and related optimal control problems. $J$. Comput. Phys., 136(1) :68-82, 1997.

[3] Michele Benzi, Gene H. Golub, and Jörg Liesen. Numerical solution of saddle point problems. Acta Numer., 14 :1-137, 2005.

[4] Michele Benzi and Valeria Simoncini. On the eigenvalues of a class of saddle point matrices. Numer. Math., 103(2) :173-196, 2006.

[5] Susanne C. Brenner and L Ridgway. Scott. The Mathematical Theory of Finite Element Methods. Springer, New York, Applied Mathematics edition, 2008. 
[6] Xiao-Chuan Cai and Marcus Sarkis. A restricted additive Schwarz preconditioner for general sparse linear systems. SIAM Journal on Scientific Computing, 21 :239-247, 1999.

[7] Lea Conen, Victorita Dolean, Rolf Krause, and Frédéric Nataf. A coarse space for heterogeneous Helmholtz problems based on the Dirichlet-toNeumann operator. J. Comput. Appl. Math., 271 :83-99, 2014.

[8] Bruno Després. Décomposition de domaine et problème de Helmholtz. C.R. Acad. Sci. Paris, 1(6) :313-316, 1990.

[9] Bruno Després. Domain decomposition method and the Helmholtz problem.II. In Second International Conference on Mathematical and Numerical Aspects of Wave Propagation (Newark, DE, 1993), pages 197-206, Philadelphia, PA, 1993. SIAM.

[10] Bruno Després, Patrick Joly, and Jean E. Roberts. A domain decomposition method for the harmonic Maxwell equations. In Iterative methods in linear algebra (Brussels, 1991), pages 475-484, Amsterdam, 1992. North-Holland.

[11] Victorita Dolean, Luca Gerardo Giorda, and Martin J. Gander. Optimized Schwarz methods for Maxwell equations. SIAM J. Scient. Comp., 31(3) :2193-2213, 2009.

[12] Victorita Dolean, Pierre Jolivet, and Nataf. An Introduction to Domain Decomposition Methods : algorithms, theory and parallel implementation. SIAM, 2015.

[13] Yalchin Efendiev, Juan Galvis, Raytcho Lazarov, and Joerg Willems. Robust domain decomposition preconditioners for abstract symmetric positive definite bilinear forms. ESAIM Math. Model. Numer. Anal., 46(5) :1175$1199,2012$.

[14] Evridiki Efstathiou and Martin J. Gander. Why restricted additive Schwarz converges faster than additive Schwarz. BIT, 43(suppl.) :945-959, 2003.

[15] Charbel Farhat, A. Macedo, and M. Lesoinne. A two-level domain decomposition method for the iterative solution of high-frequency exterior Helmholtz problems. Numer. Math., 85(2) :283-303, 2000.

[16] Juan Galvis and Yalchin Efendiev. Domain decomposition preconditioners for multiscale flows in high contrast media : reduced dimension coarse spaces. Multiscale Model. Simul., 8(5) :1621-1644, 2010.

[17] Martin J. Gander. Optimized Schwarz methods. SIAM J. Numer. Anal., 44(2):699-731, 2006.

[18] Martin J. Gander, Frédéric Magoulès, and Frédéric Nataf. Optimized Schwarz methods without overlap for the Helmholtz equation. SIAM J. Sci. Comput., 24(1) :38-60, 2002.

[19] Pascal Havé, Roland Masson, Frédéric Nataf, Mikolaj Szydlarski, Hua Xiang, and Tao Zhao. Algebraic domain decomposition methods for highly heterogeneous problems. SIAM J. Sci. Comput., 35(3) :C284-C302, 2013.

[20] F. Hecht. New development in freefem++. J. Numer. Math., 20(3-4) :251$265,2012$. 
[21] Caroline Japhet, Frédéric Nataf, and Francois-Xavier Roux. The Optimized Order 2 Method with a coarse grid preconditioner. application to convection-diffusion problems. In P. Bjorstad, M. Espedal, and D. Keyes, editors, Ninth International Conference on Domain Decompositon Methods in Science and Engineering, pages 382-389. John Wiley \& Sons, 1998.

[22] P. Jolivet, F. Hecht, F. Nataf, and C. Prud'homme. Scalable domain decomposition preconditioners for heterogeneous elliptic problems. In Proceedings of the 2013 ACM/IEEE conference on Supercomputing, SC13, pages $80: 1-80: 11$. ACM, 2013. Best paper finalist.

[23] Pierre Jolivet and Frédéric Nataf. Hpddm : High-Performance Unified framework for Domain Decomposition methods, MPI-C++ library. https ://github.com/hpddm/hpddm, 2014.

[24] Richard B Lehoucq, Danny C Sorensen, and Chao Yang. ARPACK users' guide : solution of large-scale eigenvalue problems with implicitly restarted Arnoldi methods, volume 6. SIAM, 1998.

[25] Jörg Liesen and Beresford N. Parlett. On nonsymmetric saddle point matrices that allow conjugate gradient iterations. Numer. Math., 108(4) :605$624,2008$.

[26] Pierre-Louis Lions. On the Schwarz alternating method. II. In Tony Chan, Roland Glowinski, Jacques Périaux, and Olof Widlund, editors, Domain Decomposition Methods, pages 47-70, Philadelphia, PA, 1989. SIAM.

[27] Pierre-Louis Lions. On the Schwarz alternating method. III : a variant for nonoverlapping subdomains. In Tony F. Chan, Roland Glowinski, Jacques Périaux, and Olof Widlund, editors, Third International Symposium on Domain Decomposition Methods for Partial Differential Equations, held in Houston, Texas, March 20-22, 1989, Philadelphia, PA, 1990. SIAM.

[28] Pierre-Louis Lions. On the Schwarz alternating method. III : a variant for nonoverlapping subdomains. In Tony F. Chan, Roland Glowinski, Jacques Périaux, and Olof Widlund, editors, First International Symposium on Domain Decomposition Methods for Partial Differential Equations, Philadelphia, PA, 1990. SIAM.

[29] Jan Mandel. Balancing domain decomposition. Comm. on Applied Numerical Methods, 9 :233-241, 1992.

[30] Frédéric Nataf, Francois Rogier, and Eric de Sturler. Optimal interface conditions for domain decomposition methods. Technical Report 301, CMAP (Ecole Polytechnique), 1994.

[31] Frédéric Nataf, Hua Xiang, Victorita Dolean, and Nicole Spillane. A coarse space construction based on local Dirichlet to Neumann maps. SIAM J. Sci Comput., 33(4) :1623-1642, 2011.

[32] Sergey V. Nepomnyaschikh. Mesh theorems of traces, normalizations of function traces and their inversions. Sov. J. Numer. Anal. Math. Modeling, $6: 1-25,1991$. 
[33] Roy A. Nicolaides. Deflation of conjugate gradients with applications to boundary value problems. SIAM J. Numer. Anal., 24(2) :355-365, 1987.

[34] H. A. Schwarz. Über einen Grenzübergang durch alternierendes Verfahren. Vierteljahrsschrift der Naturforschenden Gesellschaft in Zürich, 15 :272286, 1870.

[35] Nicole Spillane, Victorita Dolean, Patrice Hauret, Frédéric Nataf, Clemens Pechstein, and Robert Scheichl. Abstract robust coarse spaces for systems of PDEs via generalized eigenproblems in the overlaps. Numer. Math., 126(4) :741-770, 2014.

[36] Nicole Spillane, Victorita Dolean, Patrice Hauret, Frédéric Nataf, and Daniel Rixen. Solving generalized eigenvalue problems on the interfaces to build a robust two-level FETI method. C. R. Math. Acad. Sci. Paris, 351(5-6) :197-201, 2013.

[37] Amik St-Cyr, Martin J. Gander, and S. J. Thomas. Optimized multiplicative, additive, and restricted additive Schwarz preconditioning. SIAM J. Sci. Comput., 29(6) :2402-2425 (electronic), 2007.

[38] Andrea Toselli and Olof Widlund. Domain Decomposition Methods - Algorithms and Theory, volume 34 of Springer Series in Computational Mathematics. Springer, 2005.

[39] Xuemin Tu and Jing Li. A FETI-DP type domain decomposition algorithm for three-dimensional incompressible Stokes equations. SIAM J. Numer. Anal., 53(2) :720-742, 2015. 